FIVE YEARS EXPERIENCE WITH NCPAP AS THE STANDARD OF PRACTICE IN POLAND

J. Swietlinski ${ }^{1}$, E. Gajewska², T. Bachman ${ }^{3}$, K. Bober ${ }^{4}$, E. Helwich ${ }^{5}$, R. Lauterbach ${ }^{6}$, B. Maruszewski ${ }^{7}$, J. Szczapa ${ }^{8}$, M. Skrzypek ${ }^{9}$

${ }^{1}$ Research, IPCZD, Warszawa, ${ }^{2}$ Neonatology, "Medical University, Wroclaw, Poland, ${ }^{3}$ Mountains Community Hospital, Arrow Springs, CA, USA, ${ }^{4}$ Neonatology, Silesian Medical University, Katowice, ${ }^{5}$ Neonatology, IMiDZ, Warszawa, ${ }^{6}$ Neonatology, Jagiellonian University Medical College, Krakow, ${ }^{7}$ Cardiosurgery, IPCZD, Warszawa, ${ }^{8}$ Neonatology, Medical University, Poznan, ${ }^{9}$ Biostatistics, Silesian Medical University, Katowice, Poland

Objective: We hypothesized that nCPAP could be applied successfully across our country and that gathering of relevant data would identify factors associated with successful treatment.

Methods: We implemented a program in 2003 to establish general use of nCPAP as the standard of practice. The variable flow Infant Flow was selected as the system of choice. A registry of the baseline, course of treatment and outcome of all infants treated with nCPAP was established. In this analysis we present our experience with 11,476 neonates treated with nCPAP over the first 5 year period.

Results: $41 \%$ of the infants were between 1000 2000 grams, $14 \%$ below and $45 \%$ above. Most of the infants were treated to avoid intubation $(67 \%$ RDS, $12 \%$ other, $5 \%$ apnea), and the balance to avoid reintubation when weaning from mechanical ventilation. The percentage of infants in the weaning category decreased as elective intubation decreased. nCPAP treatment successfully avoided intubation or reintubation $84 \%$ of the time. Multivariate analysis revealed that treatment success was not only associated with diagnostic category, weight and EGA, but also Apgar score at 5 minutes, $\mathrm{pH}>7.25$ and $\mathrm{PaO} 2 / \mathrm{FiO} 2>150$. The incidence of pneumothorax was highest in infants larger than 1000 grams being treated to avoid intubation, but still only $2.2 \%$. Multivariate analysis revealed that pneumothorax in this group was associated with Apgar score at 5 minutes, $\mathrm{pH}<7.25$ and $\mathrm{PaO} 2 / \mathrm{FiO} 2<150$.

Conclusions: We successfully established nCPAP as a standard of practice in Poland. We identified baseline factors associated with bad outcomes.

\section{VOLUME GUARANTEE SETTING AND SPONTANEOUS RESPIRATORY RATE RESPONSE IN A NEONATAL ANIMAL MODEL OF RDS}

\author{
M. Sanchez Luna ${ }^{1}$, M. Santos ${ }^{2}$, F. Tendillo ${ }^{2}$
}

${ }^{1}$ Hospital General Universitario Gregorio Marañon, ${ }^{2}$ Hospital Universitario Puerta de HierroMajadahonda, Madrid, Spain

Background: The spontaneous breathing rate (SRR) response to different tidal volume setting during volume guarantee ventilation (VG) combined with flow cycled ventilation, (PSV) could represent an adequate response in keeping a constant mminute volume ventilation, and probably correlates with $\mathrm{PaCO}_{2}$ variations.

Objective: To investigate the effect of different $V_{T}$ settings in the spontaneous respiratory rate (SRR), respiratory rate/tidal volume ratio (RVR), minute volume ventilation and lung mechanics.

Design/Methods: Six newborn piglets were ventilated with a Dräger BB8000+ ventilator in PSV with spontaneous breathing and VG initially set at $4 \mathrm{ml} / \mathrm{kg}$ of $V_{T}$ and increased in 10 min intervals in 1 $\mathrm{ml} / \mathrm{kg}$ until a $V_{T}$ of $10 \mathrm{ml} / \mathrm{kg}$. with normal lungs and after artificial RDS induced with a BAL. Respiratory mechanics, heart rate and blood pressure were continuously recorded and arterial blood gas measured after each 10 min intervals.

Results: SRR, RVR and $\mathrm{PaCO}_{2}$ decreased after increase in $V_{T}$, in normal lung $(p<0.05)$. In the RDS lung, SRR, RVR and $\mathrm{PaCO}_{2}$ increased by decreasing $V_{T}$ settings $(p<0.05)$. Minute volume inversely correlated with SRR $(p<0.05)$.

Conclusions: In this animal model, during PSV with VG ventilation in normal lungs and after BAL, changes in $V_{T}$ induced inverse changes in the SRR. The SRR and RVR value could be an important guide to know if the setting $V_{T}$ during $V G$ with PSV is adequate or not. 